\title{
Study on Usability of Agricultural Product Web Page Layout Based on Eye Tracker
}

\author{
Liu lulu \\ National languages Information Technology Research \\ Institute \\ Northwest University for Nationalities \\ Lanzhou city, Gansu Province, China
}

\author{
Wan Fucheng ${ }^{*}$ \\ Key Laboratory of National Language Intelligent Processing \\ Northwest University for Nationalities \\ Gansu Province, China
}

\author{
He Xiangzhen \\ Northwest University for Nationalities \\ Key Lab of China's National Linguistic Information \\ Technology, Gansu Province, China
}

\begin{abstract}
This paper selects 30 college students as subjects, concludes of characteristics agricultural product web page layout and goods list line density, selects 3 domestic agricultural ecommerce website interfaces which is structured from top to bottom, left corner of the right type, and 3 domestic agricultural e-commerce website interfaces that the density of web page goods list respectively for $3,4,5$ as experimental material, using the eye tracker instrument of Hi_Speed1250 to records data that subjects browse the web page to produce. According to goods list layout of web page, density of goods list and so on from the experiment, analyze the characteristics and laws of browsing a goods list page interface at hotspot distribution, to explore a layout presentation of agricultural network platform which is kind of optimization. On the one hand, it can provide reference for the front of the national characteristic e-commerce sites, on the other hand, also can save the user's cognitive resources, and improve the efficiency of the selected goods.
\end{abstract}

Keywords-eye tracker experiment; agricultural e-commerce; page layout structure; line density

\section{INTRODUCTION}

With the rapid development of network technology and the economy in our country, online shopping has become a kind of fashion. A new type of online trading platform has brought new opportunities for the development of traditional agriculture. Along with the rapid increase of website information products and services as well as the increasingly complex network structure, consumers become more and more difficult to obtain the required information effectively, how to design with availability page to attract customers and increase the efficiency of customer search information is the core issue of agricultural e-commerce web design.

In recent years, with the development and popularization of eye tracking technology is used, about web usability and page layout usability research gradually increased. Users can or not get information of web pages smoothly is an important symbol of web usability[1,2]. Thus, the problem of agricultural ecommerce web page layout of the most important is the

AUTHOR: Liu Lulu (1991-), female, postgraduate, research orientation is Human-Machine Speech Interaction. This research is supported by National Science-technology Support Plan Projects (NO. 2015BAD29B01). webpage structure and density of commodity pictures putted, that is, what we should design into page structure, and we should put in the main area focus on that how many pictures in each line is the best.

\section{Web Page Layout Available Technology Based on Eye TRACKER SELECTING A TEMPLATE}

Hartson[3] thought that availability of page layout contains usability and ease of use: usefulness refers to its function, namely has useful value of particular users, can provide all kinds of related information and content services. Ease of use refers to the structure of the site to be able to meet the user's cognitive habits, good learn ability, users quickly complete required tasks to efficient, convenient, in the using process, as well as to obtain a good subjective experience.

Some scholars from abroad have earlier eye tracker experiment was applied to web usability research, Ellis etc.[4] first eye tracking measurement as an evaluation technique to evaluate a series of website usability level. Josephson verify the Noton and Stark scanning way theory by data of different kinds of eye movement that users was watching news web pages, such as advertising web and portal web images . AC Nielsen company recorded 232 users eye movement situation through eye tracking technology in the nearly thousand website, this paper puts forward the general visual laws when users browse the web page, that is F shape model[5].

Domestic also have eye tracker experiment which was applied to web usability research. Xu Weiwei[6] through the research on education web page design, found the upper area in the web page is the visitor's visual preferences section. Min-jie wang[7] eye movement experiment was applied to the electronic commerce goods settlement page usability research, and it is concluded that the electronic commerce and usability design related principles of the page. Juan-juan xu[1] etc. through the eye movement experiment studied the Jingdong and the usability of the Amazon site merchandising, discover commodity list page on top of three interest area is pictures, text, and commodity prices. BaoLiQian[8] through the eye 
movement experiment to explore the distribution and influence factors of the hotspots in screen, found that all users on hotspots in the central area of the screen, and verified the "F" hotspots.

Nowadays, characteristic agricultural products website layout structural usability research is relatively small at home; the experimental study of characteristic agricultural products website commodity list pages key factors that affect consumer purchasing behavior is less. In general of agricultural ecommerce website layout structural usability research in China is not enough in-depth and comprehensive, to be more empirical research to enrich the research results, form the demands of consumers and the user experience, to design ecommerce sites page for reference[1,9].

\section{EXPERIMENTAL METHODS}

\section{A. Experimental Equipments}

This experiment uses the German Hi - Speed1250 eye tracker instrument records the eye movement behavior of objects, eye movement instrument with high resolution camera technology, using advanced image processing operations, iViewX Hi - Speed 1250 sampling rate is up to $1250 \mathrm{Hertz}$, delay no more than 0.5 milliseconds. It is designed based on the human body function of the jaw and broad barrier-free visual areas. Subjects' head is fixed by accord with human body ergonomics jaw, and connected to the camera system. Subjects using reaction box for feedback. IViewX eye tracking computer screen through the network, a serial port or TTL and the PC stimulated [10]. It can collect indexes, such as $t$ a heat map, fixation point trajectory, fixation time, fixation point parameters and so on.

\section{B. Subjects Selected}

This experiment to choose the school undergraduate and graduate students, a total of 30 male-female, aged 20 to 30 years old, eyesight or corrected vision is normal, no color blindness or color blindness.

\section{Experimental Preparation}

\section{1) Formatting the title}

\section{a) Experimental purpose}

Through the experiment to explore college the time and hotspots when objects browse the characteristics agricultural products website, look for common web page layout structure of accord with visitor's habits.

\section{b) Experimental design}

This experiment adopts the single factor test design, the independent variables are different layout pages of agricultural products e-commerce website, there are three kinds of layout structure: up and down, the turning left around the corner, the turning right around the corner. The dependent variables are browsing time. Subjects according to their usual habit browse the web pages.

\section{c) Experimental materials}

Analysis the structure of agricultural products sales page in domestic e-commerce sites have a summary, it is concluded that the structure of the domestic agricultural e-commerce sites is mainly, the turning left around the corner, the turning right around the corner. Thus the experiment selected the Taobao New Farmers sales page divided into up and down, Jingdong Specialty Pavilion, Chinese Benefiting-farmers site turned right around the corner as the experimental material.

\section{2) Page Density Experiment of Eye Tracker}

\section{a) Experimental purpose}

Through experimental material eye movement data, to explore influence of objects' visual search with different icon sets, find out the better number of agricultural e-commerce goods list page icon sets.

\section{b) Experimental design}

The main task of experiment is information search by subjects, that is, from commodities list pages of Amazon, Jingdong, and Easy-shopping sales page to choose suitable goods which objects want to seek for.

\section{c) Experimental materials}

The materials are different layout structure pages which put 3 Amazon goods, 4 Jingdong goods, and 5 Easy-shopping goods on goods list. Affiliations of authors should be typed in italic 8-point Arial. They should be preceded by a numerical superscript corresponding to the same superscript after the name of the author concerned. Please ensure that affiliations are as full and complete as possible and include the country.

\section{Experimental Processes}

(1) Introduce objects the whole experiment process and matters needing attention;

(2) The subjects sat in front of the Hi - Speed1250 eyemovement apparatus, adjust sitting position, the distance and height from eyes to screen, and then the experimenter to calibrate vision;

(3) Object scan experiment pages, and IViewX software automatically record eye movement data;

(4)Using software of BeGaze eye movement analysis to analyse data;

Requirement: the objects must attend trial practice once before a formal test starting.

\section{ANALYSIS OF EXPERIMENTAL DATA}

\section{A. Experimental Processes}

\section{- (1) Area of interest (AOI)division}

In this experiment, the experimental materials according to the page structure is divided into 2 interest area respectively, the following Fig.:( Fig.1, Fig.2, Fig.3.)

- (2) According to the above interest area, BeGaze eye software will automatically generate related eye movement data, and then use SPSS software analyze statistical data. Remove 6 invalid data, in the remaining 24 data the first time 
and total time have shown in below tables by staring at AOI 1 , AOI 2 area.

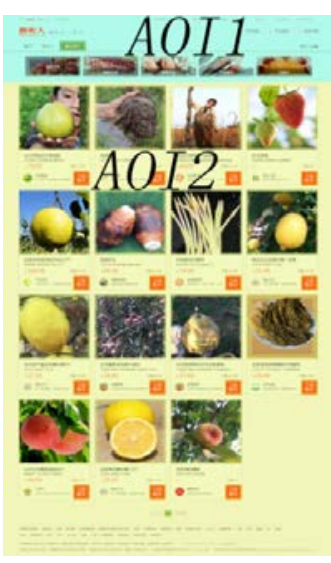

Fig.1. Taobao interested area

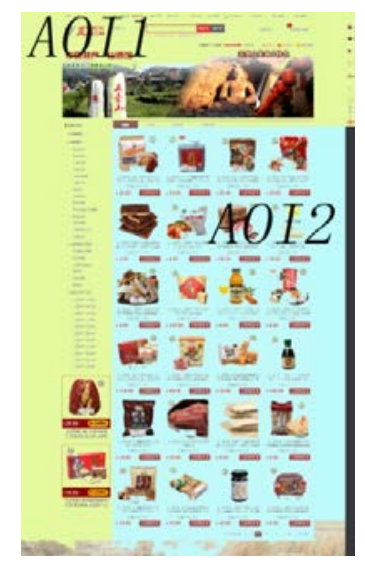

Fig.2. Jingdong interested area

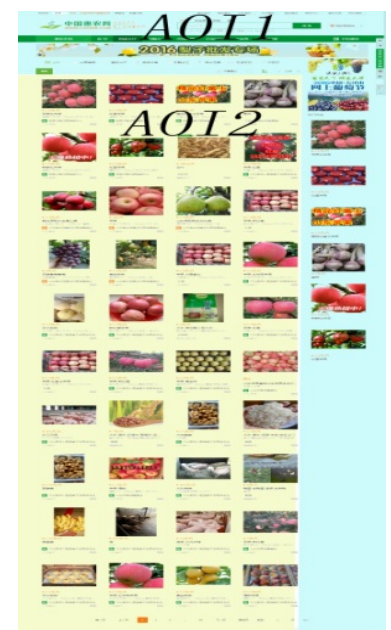

Fig.3. Chinese Benefiting-farmers site interested area

Look from the first time looking time for the area, as the page layout and navigation of AOI 1 area is first focused by the subjects, and as the main content area AOI 2 area was second focused by the subjects. Related the AOI 2, AOI 1 area is more likely to be part of the test found early. And the total focused time of AOI2 is obviously more than AOI1, illustrate AOI2 regional content is captured more attention.
There are 3 web site heat maps as follows (note: heat map according to looking time from long to short turn red, yellow, green)

From heat map we can see Taobao and Chinese Benefitingfarmers AOI 1 zone and 2 AOI area heat map is not obvious. And Jingdong heat map shape is closer to the web site of the original page layout, can guide consumers to scan pages, suited consumer's habits.

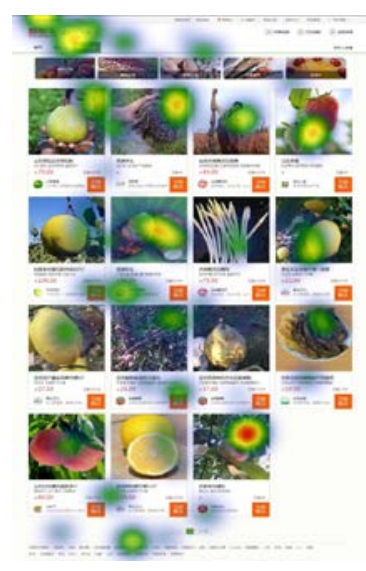

Fig.4. Taobao heat map

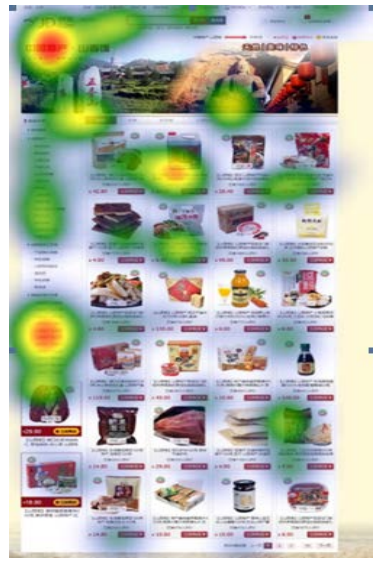

Fig.5. Jingdong heat map

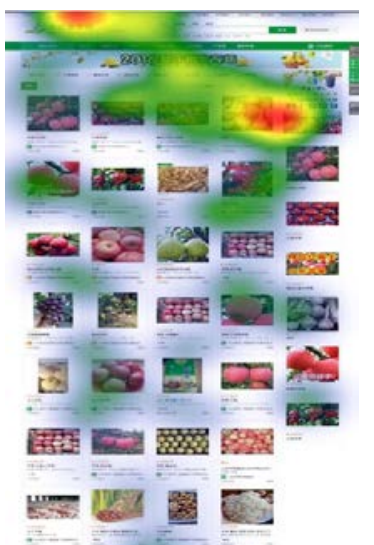

Fig.6. Chinese Benefiting-farmers heat map

TABLE I. TIME TO FIRST FIXATION

\begin{tabular}{|c|c|c|c|c|c|c|c|}
\hline \multirow{2}{*}{$\begin{array}{c}\text { Interest } \\
\text { area }\end{array}$} & \multirow{2}{*}{$\begin{array}{c}\text { The } \\
\text { number }\end{array}$} & \multicolumn{2}{|c|}{ Taobao New Farmers } & Jingdong Specialty Pavilion & \multicolumn{2}{c|}{ Chinese Benefiting-farmers } \\
\cline { 3 - 8 } & & average & standard deviation & average & standard deviation & average & standard deviation \\
\hline AOI1 & 24 & 1000 & 17 & 987 & 16.4 & 1035 & 18.6 \\
\hline AOI2 & 24 & 4944 & 14.3 & 9826 & 13.5 & 7410 & 15.8 \\
\hline
\end{tabular}

TABLE II. FIXATION DURATION OF TOTAL

\begin{tabular}{|c|c|c|c|c|c|c|}
\hline \multirow{2}{*}{$\begin{array}{c}\text { Interest } \\
\text { area }\end{array}$} & \multicolumn{2}{|c|}{ Taobao New farmers } & \multicolumn{2}{c|}{ Jingdong Specialty Pavilion } & \multicolumn{2}{c|}{ Chinese Benefiting-farmers } \\
\cline { 2 - 6 } & Staring at the total time & average & Look at the total time & average & Look at the total time & average \\
\hline AOI1 & 8973 & 206.2 & 10917.7 & 264.1 & 10063.3 \\
\hline AOI2 & 17081 & 421.6 & 21057.1 & 649.5 & 17938.4 \\
\hline
\end{tabular}


From the above table 1 and table 2 and heat map Fig., we can see that all 3 web page layout and content areas are reasonable, conform to the visitors to browse the web order, first subjects gazed at AOI1 area significantly ahead of time than AOI2 area, look at the total time of AOI2 area is longer than AOI1 area. Through the analysis of the data of experiment, we found the eye first movement focused on the top left corner of the page, the subjects are used to from left to right, from top to bottom in the browse pages, but most of the design of the computer application used to from left-up to right to operate[11] . Therefore, when design the user page interface, should try to keep this format, respect the habit of learners. Important elements should be paid attention to when design page to focus on these key areas, to ensure that your attention to this area, lead to the consumer.

\section{B. Analysis of Page Density Data}

Experimental results to remove 5 invalid data, effective experimental data obtained a total of 25. Through BeGaze software to analyze automatically, generate related eye movement data, then use SPSS software to analyze statistical data, get data of search for specific target completion average time and heat maps in 3site pages, as shown in table3, Fig. 7, Fig. 8 and Fig. 9:

TABLE III. THE COMPLETION TIME (UNIT: MS)

\begin{tabular}{|c|c|c|c|}
\hline Website & number & sum & average \\
\hline Amazon & 25 & 465305 & 18612.2 \\
\hline $\begin{array}{c}\text { Jingdong Specialty } \\
\text { Pavilion }\end{array}$ & 25 & 784322.5 & 31372.9 \\
\hline Easy-shopping & 25 & 517125 & 20685 \\
\hline
\end{tabular}

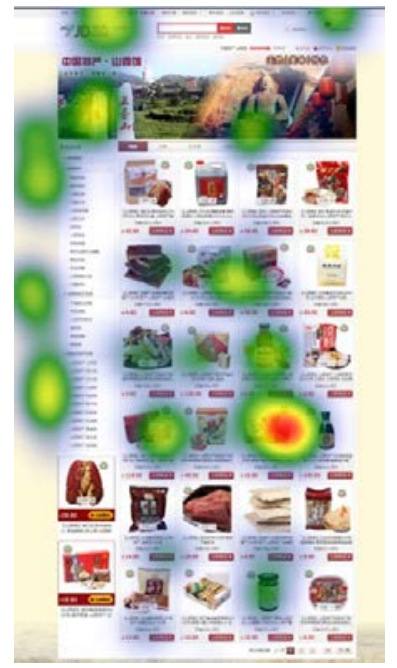

Fig.7. Jingdong search heat map

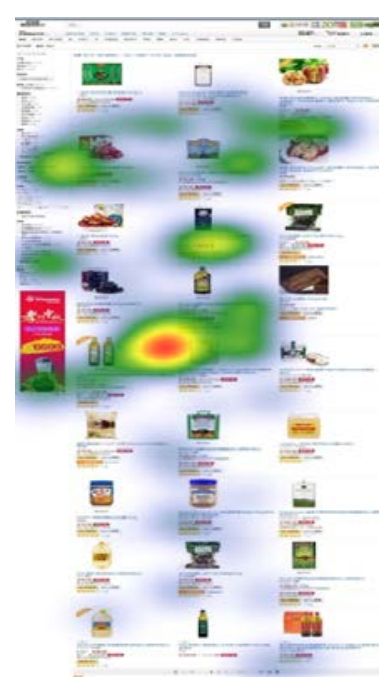

Fig.8. Amazon search heat map

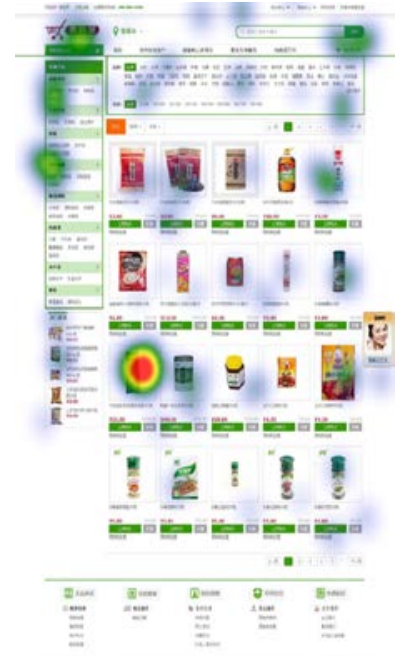

Fig.9. Easy-shopping search heat map

According to the data that the density of 3 site pages goods list influence on page layout, we can analyse that subjects complete the find out the targets of goods with the time $18612.2 \mathrm{~ms}$, 31372.9ms, 20685ms respectively. That is, Easyshopping takes the shortest completion time, Amazon takes the completion time second, Jingdong completed the longest time.

From 3 site page heat map, they can be seen intuitive, degree of concise in Jingdong, Amazon, Easy-shopping page come to reduce, subjects stay for a long time in the flaring picture place, each picture of Easy-shopping which page layout is concise has been fixed, but it costs the shortest time, and the efficiency is highest. That means, Easy-shopping's web page most conforms to the visitor's browsing habits, try to be visual pressure to a minimum.

Experimental results show that, the task of no time limit on the search specified goods can be completed on 3 pages, but finish time has significant difference, degree of concise and commodity page will also to a certain extent, affect the result of the experiment. Thus we find in providing enough information at the same time, the number of icons and interface design should be simple, can't bring too much visual and memory burden to users. For example, the concept of commodity icon model should match the consumers' cognitive psychology; the words of the text should be completely, shortly, easily to understand as far as possible; pay attention to the page layout and so on.

\section{CONCLUSIONS}

This research chose the agricultural e-commerce sites as targets, through the eye tracker experiment of five commodities list page layout structure of domestic mainstream agricultural e-commerce sites, and pages line density, to analyze relative research. The layout from the heat map, Jingdong to guide the visitors to browse the whole page, heat region is much closer to the original web page layout. Generally speaking, make a left turn type layout more accord with the public browsing habits.

Web page line distribution density of Easy-shopping is easier to identify, the pictures for the goods in the same format have significant differences of staring time, show that 
consumers need to choose goods pictures can have a more intuitive understanding of judgment.

\section{ACKNOWLEDGMENT}

This research is supported by the Fundamental Research Funds for the Central Universities (NO. Yxm2015190).

\section{REFERENCES}

[1] Juanjuan Xu, Qian Xuchao, Zhao Kai, Du Kebo. B2C website display availability of eye Tracker assessment [J]. Journal of psychological techniques and applications, 2014, 10: 13-17. (In Chinese)

[2] Fang Kai, Duan Qijun. Based on eye tracker experiment of mobile phone interface usability research [J]. Science and technology, 2015, 06: $13+31$. (In Chinese)

[3] Hartson H R. Human-computer interaction: interdisciplinary roots and trends $[\mathrm{J}]$. The Journal of System and Software, 1998, 43(2):103-118.

[4] Ellis S, Candera R, Misner Jetal. Windows to the Soul? What Eye Movements Tell Us about Software Usability.[C]Proceedings of the
Usability Professionals’ Association. Washington, DC: UPA Press, 1998: 151-178.

[5] Nielsen J. F-shaped pattern for reading web content[J]. Alertbox: Current Issues in Web Usability (retrieved 31 Oct 2008 from http://www. useit. com/alertbox/reading_ pattern. html), 2006.

[6] Xu Weiwei. Based on the education of the law of the line of sight web structure design research [D]. Ningbo university, 2012. (In Chinese)

[7] Wang Minjie, Zhu Longfei, Cui Zhenghu, Cai Leili, Hu Fengpei. Ecommerce site page layout to the settlement of eye movement research [J]. Journal of human ergonomics, 2013,04:33-37. (In Chinese)

[8] BaoLiQian. Web browsing screen visual hotspots in the regional distribution of research [D]. Central China normal university, 2015. (In Chinese)

[9] Chong Shuqing, Fan Jun jing, Wang Yecheng. Eye movement technology in the application of website usability inspection research [J]. Journal of design, 2015, 23:132-133. (In Chinese)

[10] Zhu Shengyin. Tibetan and remote education based on eye movement instrument user interface design research [D]. Northwest university for nationalities, 2012. (In Chinese)

[11] Hu Fangqin. Interested in screen area based on line of sight detection track [D]. Zhejiang university, 2015. (In Chinese) 